\title{
Visuospatial Memory Deficits Emerging During Nicotine Withdrawal in Adolescents with Prenatal Exposure to Active Maternal Smoking
}

\author{
Leslie K Jacobsen*, I,2,3, Theodore A Slotkin ${ }^{4}$, Michael Westerveld ${ }^{5,6}$, W Einar Mencl ${ }^{3}$ and Kenneth R Pugh ${ }^{2,3}$ \\ 'Department of Psychiatry, Yale University School of Medicine, New Haven, CT, USA; ${ }^{2}$ Department of Pediatrics, Yale University School of \\ Medicine, New Haven, CT, USA; ${ }^{3}$ Haskins Laboratories, New Haven, CT, USA; ${ }^{4}$ Department of Pharmacology and Cancer Biology, Duke \\ University Medical Center, Durham, NC, USA; ${ }^{5}$ Department of Neurosurgery, Yale University School of Medicine, New Haven, CT, USA; \\ ${ }^{6}$ Department of Child Study, Yale University School of Medicine, New Haven, CT, USA
}

\begin{abstract}
Active maternal smoking during pregnancy elevates the risk of cognitive deficits and tobacco smoking among offspring. Preclinical work has shown that combined prenatal and adolescent exposure to nicotine produces more pronounced hippocampal changes and greater deficits in cholinergic activity upon nicotine withdrawal than does prenatal or adolescent exposure to nicotine alone. Few prior studies have examined the potential modifying effects of gestational exposure to active maternal smoking on cognitive or brain functional response to tobacco smoking or nicotine withdrawal in adolescents. We examined visuospatial and verbal memory in 35 adolescent tobacco smokers with prenatal exposure to active maternal smoking and 26 adolescent tobacco smokers with no prenatal exposure to maternal smoking who were similar in age, educational attainment, general intelligence, and baseline plasma cotinine. Subjects were studied during ad libitum smoking and after $24 \mathrm{~h}$ of abstinence from smoking. A subset of subjects from each group also underwent functional magnetic resonance imaging while performing a visuospatial encoding and recognition task. Adolescent tobacco smokers with prenatal exposure experienced greater nicotine withdrawal-related deficits in immediate and delayed visuospatial memory relative to adolescent smokers with no prenatal exposure. Among adolescent smokers with prenatal exposure, nicotine withdrawal was associated with increased activation of left parahippocampal gyrus during early recognition testing of visuospatial stimuli and increased activation of bilateral hippocampus during delayed recognition testing of visuospatial stimuli. These findings extend prior preclinical work and suggest that, in human adolescent tobacco smokers, prenatal exposure to active maternal smoking is associated with alterations in medial temporal lobe function and concomitant deficits in visuospatial memory.

Neuropsychopharmacology (2006) 31, I550- I56I. doi:I 0.1038/sj.npp. 130098 I; published online 7 December 2005
\end{abstract}

Keywords: adolescence; nicotine; brain development; maternal smoking; visuospatial memory; encoding; retrieval; recognition memory

\section{INTRODUCTION}

Epidemiologic data suggest that more than half of tobaccosmoking women continue to smoke through their pregnancies, resulting in at least a half a million births per annum of infants prenatally exposed to tobacco smoke in the United States (Ebrahim et al, 2000; Martin et al, 2005). Prospective clinical studies have linked prenatal exposure to tobacco smoke to reductions in motor and verbal comprehension scores in children assessed at 13 months of age (Gusella and

* Correspondence: Dr LK Jacobsen, Psychiatry and Pediatrics, Yale University School of Medicine, 2 Church Street South, Suite 207, New Haven, CT 06519, USA, Tel: + I 203764 8480, Fax: + I 203764 8484, E-mail: leslie.jacobsen@yale.edu

Received I6 May 2005; revised 13 September 2005; accepted 13 October 2005

Online publication: 2 I October 2005 at http://www.acnp.org/citations/ Npp I02 I 05050322/default.pdf
Fried, 1984), reductions in measures of language development, and general intellectual function in children assessed at 3, 4, 5, and 6 years of age (Fried and Watkinson, 1990; Fried et al, 1992), deficits in verbal and nonverbal learning and memory in preadolescents (Cornelius et al, 2001), and reductions in general intelligence in children assessed between 9 and 16 years of age (Fried et al, 1998, 2003).

Preclinical studies have provided strong evidence that the disruptive effects of nicotine on brain development contribute significantly to these deleterious effects of gestational exposure to tobacco smoke (Slotkin, 2004). Nicotine, the primary psychoactive component of tobacco smoke, binds to nicotinic acetylcholine receptors (nAChRs), which become detectable in embryonic development shortly before neurulation (Atluri et al, 2001; Schneider et al, 2002). Acetylcholine plays a critical developmental role in brain maturation (Lauder and Schambra, 1999; Weiss et al, 1998; Slotkin, 2004), initially promoting cell division, and 
subsequently promoting the switch from replication to differentiation (Slotkin, 1998, 1999). In rodents, inappropriate stimulation of $\mathrm{nAChRs}$ by nicotine during prenatal development produces reductions in brain cell number, size, and surface area (Roy et al, 1998; Slotkin, 1998, 1999), prolonged reductions in cholinergic neural transmission (Zahalka et al, 1992; Navarro et al, 1989), and alters the subsequent response to nicotine challenge (Navarro et al, 1988; Seidler et al, 1992; Levin et al, 1993, 1996).

Recent work with a rodent model of adolescent nicotine exposure provided evidence that vulnerability to the developmentally disruptive effects of nicotine extends postnatally into adolescence (Slotkin, 2002). In doses designed to model typical human exposures, nicotine treatment of rats during adolescence altered cell numbers in cortex, midbrain, and hippocampus (Trauth et al, 2000), and produced prolonged suppression of cholinergic neural activity and responsiveness (Abreu-Villaca et al, 2003; Trauth et al, 2001). Consistent with this preclinical work, human adolescent smokers were recently found to have impairments in accuracy of verbal working memory performance relative to nonsmokers who were similar in age, gender, educational attainment, and duration of prenatal exposure to active maternal smoking (Jacobsen et al, 2005). Performance decrements were more severe with earlier age at the onset of smoking. In adult humans, cessation of regular tobacco smoking disrupts memory (Pineda et al, 1998; West and Hack, 1991) and attention (Shiffman et al, 1995; Snyder et al, 1989), while administration of nicotine, or resumption of tobacco smoking, reverses these withdrawal-related deficits (Foulds et al, 1996; Krebs et al, 1994; Pineda et al, 1998). Consistent with these observations in adults, memory deficits worsened in adolescent tobacco smokers during nicotine withdrawal (Jacobsen et al, 2005).

Rates of tobacco smoking and nicotine dependence among offspring prenatally exposed to active maternal smoking are elevated (Buka et al, 2003; Kandel et al, 1994; Griesler et al, 1998; Cornelius et al, 2000). Recent data from the US National Comorbidity Survey indicate that onset of daily smoking is concentrated between the ages of 15 and 20 years, suggesting that adolescence is a critical period for progression from initial to regular tobacco use (Breslau et al, 2001). Adolescence is also a period of continued brain development, with ongoing neuroproliferation, apoptosis, synaptic rearrangement, and maturation of central cholinergic systems (Bayer, 1983; Bayer et al, 1982; Huttenlocher, 1990; Matthews et al, 1974; Nadler et al, 1974; Zahalka et al, 1993). Rodent models developed to investigate the effects of prenatal nicotine exposure on response to nicotine administration during adolescence have shown that prenatal nicotine exposure reduces the upregulation of nAChRs induced by adolescent nicotine exposure, produces cholinergic hypoactivity during nicotine withdrawal in adolescence (Abreu-Villaca et al, 2004b), and magnifies the effects of adolescent nicotine exposure on indices of cell number, size, and surface area (Abreu-Villaca et al, 2004a).

To the best of our knowledge, no prior study has examined the potential modifying effects of gestational exposure to active maternal smoking on cognitive or brain functional response to tobacco smoking or nicotine with- drawal in human adolescents or adults. In the present study, verbal and visuospatial memory were examined in adolescent regular tobacco smokers with and without prenatal exposure to active maternal smoking, during ad libitum smoking and during smoking abstinence.

Prior work has shown that reducing cholinergic neurotransmission disrupts memory (Rosier et al, 1998, 1999; Mewaldt and Ghoneim, 1979). Given that nicotine withdrawal induces cholinergic hypoactivity in adolescent rodents with gestational exposure to nicotine (AbreuVillaca et al, 2004b), we anticipated that nicotine withdrawal-related disruption of memory would be greater in adolescents with prenatal exposure to active maternal smoking than in adolescents without such exposure. Medial temporal lobe structures, including hippocampus and parahippocampal gyrus, provide critical support for both encoding and retrieval of explicit memory (Cabeza et al, 2001; Eichenbaum et al, 1996; Squire, 1992), and evidence suggests that hippocampal cholinergic neurotransmission modulates the efficacy of encoding (Sperling et al, 2002). A subset of subjects were studied using functional magnetic resonance imaging during performance of a task designed to permit separate examination of neural circuits mediating encoding and recognition memory. Prior work has shown that enhanced cholinergic neurotransmission increases the selectivity of perceptual processing during encoding, thereby reducing demand on, and activation of, regions involved in higher-order processes (Furey et al, 2000). Given evidence that nicotine withdrawal induces cholinergic hypoactivity in adolescent rodents with gestational exposure to nicotine (Abreu-Villaca et al, 2004b), we hypothesized that efficacy of encoding would be impaired during nicotine withdrawal in adolescents with prenatal exposure to active maternal smoking, and that this would increase the demand on and activation of hippocampus during recognition memory testing.

\section{METHODS}

\section{Participants}

In total, 35 adolescent daily tobacco smokers with prenatal exposure to active maternal smoking were compared with 26 adolescent daily tobacco smokers with no prenatal exposure to active maternal smoking. All participants were recruited from the community by newspaper advertisement and flyer distribution in the context of an ongoing study of adolescent tobacco use. All subjects were free of medical and psychiatric illness and substance abuse or dependence disorders, other than nicotine dependence, as determined by physical exam and structured clinical interview using the Schedule for Affective Disorders and Schizophrenia for School Aged Children (Kaufman et al, 1996) performed at initial screening. Detailed drug use history was obtained using the Comprehensive Addiction Severity Index for Adolescents (Meyers et al, 1995). Subjects were required to abstain from all drug use for 1 month prior to assessment and from alcohol for $48 \mathrm{~h}$ prior to assessment. Abstinence from substance use prior to assessment was confirmed by urine toxicology screen.

At initial screening, general intelligence was estimated using the Kaufman Brief Intelligence Test (Bowers and 
Pantle, 1998), reading achievement was estimated using the Word Attack subtest of the Woodcock-Johnson Revised Test of Achievement, and self-reported symptoms of depression, anxiety, stressful life events, and inattention were assessed using the Beck Depression Scale (Beck et al, 1961), the Multidimensional Anxiety Scale for Children (March et al, 1997), the Life Events Questionnaire (Masten et al, 1994), and the Conners Adolescent Self-Report Scale (Conners, 1998), respectively. Prenatal exposure was assessed at the time of initial screening using a semistructured interview of the parents regarding the average number of cigarettes smoked per day by the mother during each trimester of her pregnancy with the subject. The mother's consumption of other substances and alcohol during the pregnancy, and the rate of smoking by other persons living in the home during the pregnancy (prenatal environmental exposure), were also assessed during this interview.

Parental consent was obtained for subjects 17 years of age and younger. This study was approved by the Yale University School of Medicine Human Investigation Committee. Subjects provided written assent or, for 18 year-olds, consent for study participation.

\section{Procedure}

All subjects were tested twice, during ad libitum smoking and after $24 \mathrm{~h}$ of abstinence from tobacco. Test sessions were conducted in the late afternoon for all subjects. Prior to the abstinence test session, subjects were instructed to refrain from using any tobacco products for $24 \mathrm{~h}$. Subjects were told what time to stop smoking and were provided reminders and encouragement to comply with the tobacco restriction by telephone contact. To avoid the emergence of nicotine withdrawal during the smoking test session, subjects were permitted to smoke their own brand of cigarettes during a break midway through the test session. Order of smoking and abstinence test sessions was randomized. At the beginning of each test session, nicotine withdrawal was assessed using the Minnesota Nicotine Withdrawal Scale (Hughes and Hatsukami, 1998), and tobacco craving was assessed using the Tiffany Scale for Smoking Urges (Tiffany and Drobes, 1991) and the Shiffman Jarvik Scale (Shiffman and Jarvik, 1976).

During each test session, blood or, in 21 subjects who declined phlebotomy, saliva was obtained to measure nicotine and cotinine, the primary metabolite of nicotine, using gas chromatography (Jacob et al, 1981). The proportion of subjects declining phlebotomy did not differ across groups $\left(\chi^{2}=1.2, p=0.3\right)$. To reduce food contamination, saliva was collected after subjects rinsed their mouths with water. Saliva and plasma were frozen at $-30^{\circ} \mathrm{C}$ immediately following collection. All samples were shipped on dry ice to the Clinical Pharmacology Laboratory of the University of California, San Francisco, where they were assayed for nicotine and cotinine. Expired air carbon monoxide (CO), a product of combustion, was also measured at each test session (Micro CO meter: Micro Direct, Auburn, ME). While examiners were not blind to prenatal exposure status of the subjects, they were blind to the hypotheses under study.

\section{Assessment of Cognitive Performance}

Visuospatial memory was assessed using the Brief Visuospatial Memory Test-Revised (BVMT-R) (Benedict et al, 1996), a figural learning task. For this task, subjects view a stimulus page with six geometric figures on it. After $10 \mathrm{~s}$, the stimulus page is removed and subjects must draw as many of the figures as possible in the correct location. This procedure is repeated twice for a total of three administrations (immediate recall trials), and is followed by a $25-\mathrm{min}$ delay interval during which the subject engages in verbal tasks. After the 25-min delay, the subject is asked once again to draw from memory as many of the figures as possible in the correct location (delayed recall trial). Following the delayed recall trial, a recognition test is administered during which the subject views 12 geometric figures in sequence, including the six figures learned during the immediate recall trials. The subject indicates whether each figure is old or new. Scores derived included total immediate recall (the sum of scores obtained during the three immediate recall trials), delayed recall, and recognition hits (the number of true positives on the recognition task). Verbal memory was assessed using the Hopkins Verbal Learning Test-Revised (HVLT-R) (Shapiro et al, 1999), an auditory word list learning task whose structure is identical to that of the BVMT-R. During the immediate recall trials of the HVLT-R, the subject is read a list of 12 words and is asked to repeat as many of them as possible after each of three readings. Delayed recall is tested after a 25-min interval, during which the subject is engaged in nonverbal tasks. Recognition memory is tested using a randomized list of 24 words that includes the 12 words learned during the immediate recall trials. After each word is read, the subject indicates whether each word is old or new. As with the BVMT-R, the HVLT-R includes measures of total immediate recall, delayed recall, and recognition hits. To minimize item-related practice effects (Benedict and Zgaljardic, 1998), different forms of the HVLT-R and BVMT-R were administered at each of the two testing sessions.

\section{Assessment of Brain Function}

To explore the effects of prenatal exposure to active maternal smoking on the functioning of neural-circuitrymediating components of mnemonic processing, seven subjects with prenatal exposure and six subjects with no prenatal exposure also underwent fMRI scanning while performing a modified version of a previously described task (Shaywitz et al, 1999; Mencl et al, 2000) that incorporated a mixed design, including block and eventrelated features (Donaldson et al, 2001; Otten et al, 2002; Visscher et al, 2003), to permit separate assessment of neural-circuitry-mediating encoding, rehearsal, and early and delayed recognition of verbal and nonverbal stimuli. Imaging and assessment of verbal and visuospatial memory were performed during the same test session.

The subgroup of adolescent tobacco smokers with and without prenatal exposure to tobacco smoke, who underwent fMRI scanning and were selected randomly from the larger group and did not differ in age (mean \pm standard deviation (SD): prenatally exposed $=16.6 \pm 1.2$ years, no 
prenatal exposure $=17.0 \pm 0.9$ years), gender (all prenatally exposed subjects were females, four subjects with no prenatal exposure were female), years of education (prenatally exposed $=10.0 \pm 1.5$ years, no prenatal exposure $=$ $10.5 \pm 0.8$ years), years of parent education (prenatally exposed $=13.8 \pm 1.7$ years, no prenatal exposure $=13.8 \pm 1.7$ years), consumption of tobacco (number of cigarettes smoked per day: prenatally exposed $=11.2 \pm 6.4$, no prenatal exposure $=9.1 \pm 4.2$ ), alcohol (number of drinks consumed per week: prenatally exposed $=2.0 \pm 4.4$, no prenatal exposure $=0.2 \pm 0.2$ ), and cannabis (lifetime episodes of cannabis use: prenatally exposed $=130 \pm 169$, no prenatal exposure $=201 \pm 286)$, symptoms of nicotine dependence (FTND score: prenatally exposed $=3.1 \pm 2.6$, no prenatal exposure $=3.5 \pm 2.5$ ), general intelligence (KBIT composite score: prenatally exposed $=94.0 \pm 7.1$, no prenatal exposure $=91.8 \pm 6.8$, reading achievement $(\mathrm{WJR}$ Word Attack subtest standard score: prenatally exposed $=102.6 \pm 16.0$, no prenatal exposure $=105.7 \pm 15.0$ ), or symptoms of anxiety (MASC score: prenatally exposed $=$ $39.7 \pm 15.1$, no prenatal exposure $=32.3 \pm 6.0$ ), depression (Beck Depression score: prenatally exposed $=7.1 \pm 6.1$, no prenatal exposure $=8.0 \pm 6.3$ ), or inattention (Conners Adolescent Self-Report Scale score: prenatally exposed = $17.4 \pm 10.0$, no prenatal exposure $=14.7 \pm 12.2$ ) at the initial screening visit. Prenatally exposed subjects included three subjects with exposure restricted to the first trimester and four subjects with exposure throughout gestation.

Each imaging run consisted of four cycles, the components of which included rest, encode, rehearse, and forced choice recognition, each of which were $20 \mathrm{~s}$ long. Verbal and nonverbal cycles alternated during each run. The structure of the nonverbal cycles is schematically depicted in Figure 1. During the nonverbal cycle encode phase, subjects saw six characters from South Asian languages (Tamil, Marathi, Bengali, Telugi, Hindi, Gujarati, Sanskrit, Kannada, and Gurmukhi) sequentially for $3.333 \mathrm{~s}$ each, followed by a $20-\mathrm{s}$ rehearsal period during which subjects were instructed to remember the words just presented. During forced choice recognition, subjects saw three familiar characters and three new characters, with the sequence of old and new

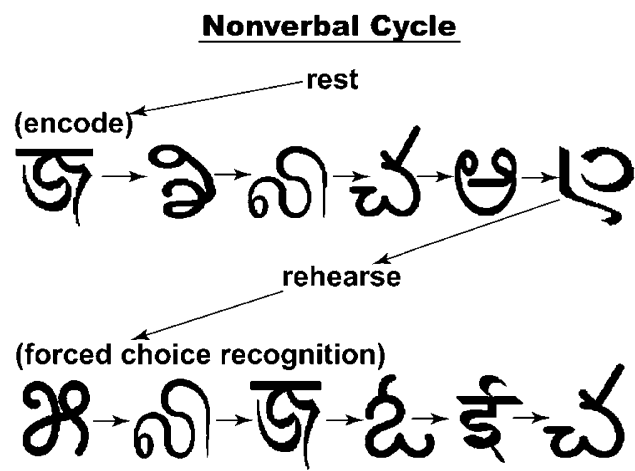

Figure I Schematic representation of the structure of the nonverbal cycles during $\mathrm{fMRI}$ scanning. During the nonverbal cycle encode phase, subjects saw six characters from South Asian languages sequentially for $3.333 \mathrm{~s}$ each, followed by a 20-s rehearsal period. During forced choice recognition, subjects saw three familiar characters and three new characters, with the sequence of old and new randomized across recognition blocks and runs. randomized across recognition blocks and runs. Subjects indicated with a button press whether each character was old (seen during encoding) or new. Verbal cycles were identical in design to the nonverbal cycles, except that stimuli were monosyllabic English words. Each block type began with a visual prompt, reminding subjects to rest, study (encode), rehearse, or test (forced choice recognition). Each run was 5 min $40 \mathrm{~s}$ long. After six runs, a sagittal anatomic MP-RAGE three-dimensional image was obtained. This was followed by two runs in which delayed recognition memory was assessed. Each of these last two runs consisted of five cycles that included a 20-s rest block followed by a 20 -s verbal recognition and a 20-s nonverbal recognition block. The order of verbal and nonverbal recognition blocks was randomized across cycles. Each delayed recognition block consisted of three stimuli presented during the encoding blocks, but not the recognition blocks during the first six runs, and three new stimuli. Subjects again indicated whether each stimulus was old or new with a button press. Due to time constraints, only stimuli presented during encoding blocks within the first five runs were included as targets in the final two (delayed recognition) runs. The time duration between the end of the fifth run and the beginning of the seventh run was $16.1 \pm 1.9$ min.

Subjects were scanned twice, during ad libitum smoking and after $24 \mathrm{~h}$ of abstinence from tobacco products, and the order of smoking and abstinence scans was randomized. Subjects were scanned using a 3.0-T Siemens Trio MRI system. Axial oblique T1-weighted anatomic images were acquired parallel to the AC/PC line using a FLASH sequence (25 contiguous slices, slice thickness $=5 \mathrm{~mm}$, $\mathrm{TE}=2.47 \mathrm{~ms}, \quad \mathrm{TR}=300 \mathrm{~ms}, \mathrm{FA}=60^{\circ}$, matrix $=256 \times 256$ pixels, $F O V=20 \mathrm{~cm}^{2}$ ). Epibold functional images were acquired in the same relative slice locations using a singleshot, gradient echo pulse sequence $(\mathrm{TE}=30 \mathrm{~ms}, \mathrm{TR}=$ $2000 \mathrm{~ms}, \mathrm{FA}=80^{\circ}$, matrix $=64 \times 64$ pixels, $\mathrm{FOV}=20 \mathrm{~cm}^{2}$ ). In all, 170 images were acquired per slice per run. The highresolution anatomic scan was acquired using a sagittal MPRAGE sequence $\left(\mathrm{TE}=3.66 \mathrm{~ms}, \mathrm{TR}=2530 \mathrm{~ms}, \mathrm{FA}=7^{\circ}\right.$, $\mathrm{FOV}=256 \times 256$ pixels, slice thickness $=1 \mathrm{~mm}, 176$ slices, $\mathrm{NEX}=1$ ).

\section{Data Analysis}

Accuracy of task performance during scanning was assessed by dividing the number of correct responses by the total number of trials (proportion correct). Data were analyzed using linear mixed-effects regression analysis implemented in SPlus (Insightful Coorporation, Seattle, WA) with subject modeled as a random effect. Models that included fixed effects for group (prenatally exposed $v s$ no prenatal exposure), smoking condition, and the interaction between group and smoking condition were estimated. The potential confounding effects of group differences in gender, years of parental education, environmental tobacco smoke exposure during gestation, rate of smoking, and severity of nicotine dependence (FTND score) were controlled by entering these variables in the regression models. Effects of trimesters of exposure to active maternal smoking were examined by comparing subjects exposed for one trimester to those exposed for three trimesters, using regression models that 
included fixed effects for trimesters of exposure (one or three), smoking condition, and the interaction between trimesters of exposure and smoking condition.

Previous work has shown that salivary concentrations of cotinine in youth are, on average, $20 \%$ higher than plasma concentrations of cotinine (Jarvis et al, 2003). Therefore, salivary cotinine concentrations were multiplied by a factor of 0.8 before combining them with measures of cotinine from plasma. Plasma nicotine concentrations were estimated from salivary nicotine concentrations using a previously described power model shown to produce good correlations between plasma and salivary nicotine concentrations (Teneggi et al, 2002). The estimated plasma nicotine concentration less than $2 \mathrm{ng} / \mathrm{ml}$ prior to the abstinence test session was accepted as evidence of abstinence from tobacco use.

Image analysis was performed using software written in Matlab (MathWorks, Natick, MA). Prior to statistical analysis, images were corrected for slice acquisition time, motion corrected for three translational directions, and for the three possible rotations using SPM 99 (Friston et al, 1995), and then spatially filtered using a Gaussian filter of full-width at half-maximum (FWHM) of $3.125 \mathrm{~mm}$. Individual subject maps of activation associated with transient processes (time-locked responses evoked by the encoding and recognition trials) and sustained processes (average activation levels during the self-guided rehearsal blocks) were generated using regression-based methods implemen- ted within the general linear model (GLM). Transient effects were coded in the GLM with a reference hemodynamic response function modeled as a gamma function (tau $=0.9$, $n=4$, delay $=2.3$; peaking at $5 \mathrm{~s}$ post-stimulus) (Boynton et al, 1996). The sustained effects of rehearsal were similarly coded by convolving a block-length boxcar function with this gamma function (Boynton et al, 1996; Visscher et al, 2003). Prior to across-subjects comparisons, fMRI data were spatially normalized to standard stereotactic space using an affine transformation (Duncan et al, 2004). Group by smoking condition interaction effects on task-related activation were assessed using voxelwise mixed-model repeated-measures analysis of variance (ANOVA) (Woods, 1996; Kirk, 1982; Holmes and Friston, 1998), with group assignment as a between-subjects variable, smoking condition as a within-subjects variable, and subject treated as random effect within each group. A voxel threshold of $\alpha=0.001$ and a cluster threshold of eight contiguous significant voxels were applied.

\section{RESULTS}

Demographic, clinical, and cognitive characteristics of both groups are presented in Table 1.

The groups did not differ in age, years of education, consumption of alcoholic beverages or cannabis, general intelligence, reading achievement, symptoms of depression

Table I Demographic, Clinical and Cognitive Characteristics of Adolescent Tobacco Smokers: 35 Subjects With and 26 Subjects Without Prenatal Exposure to Active Maternal Smoking ${ }^{a}$

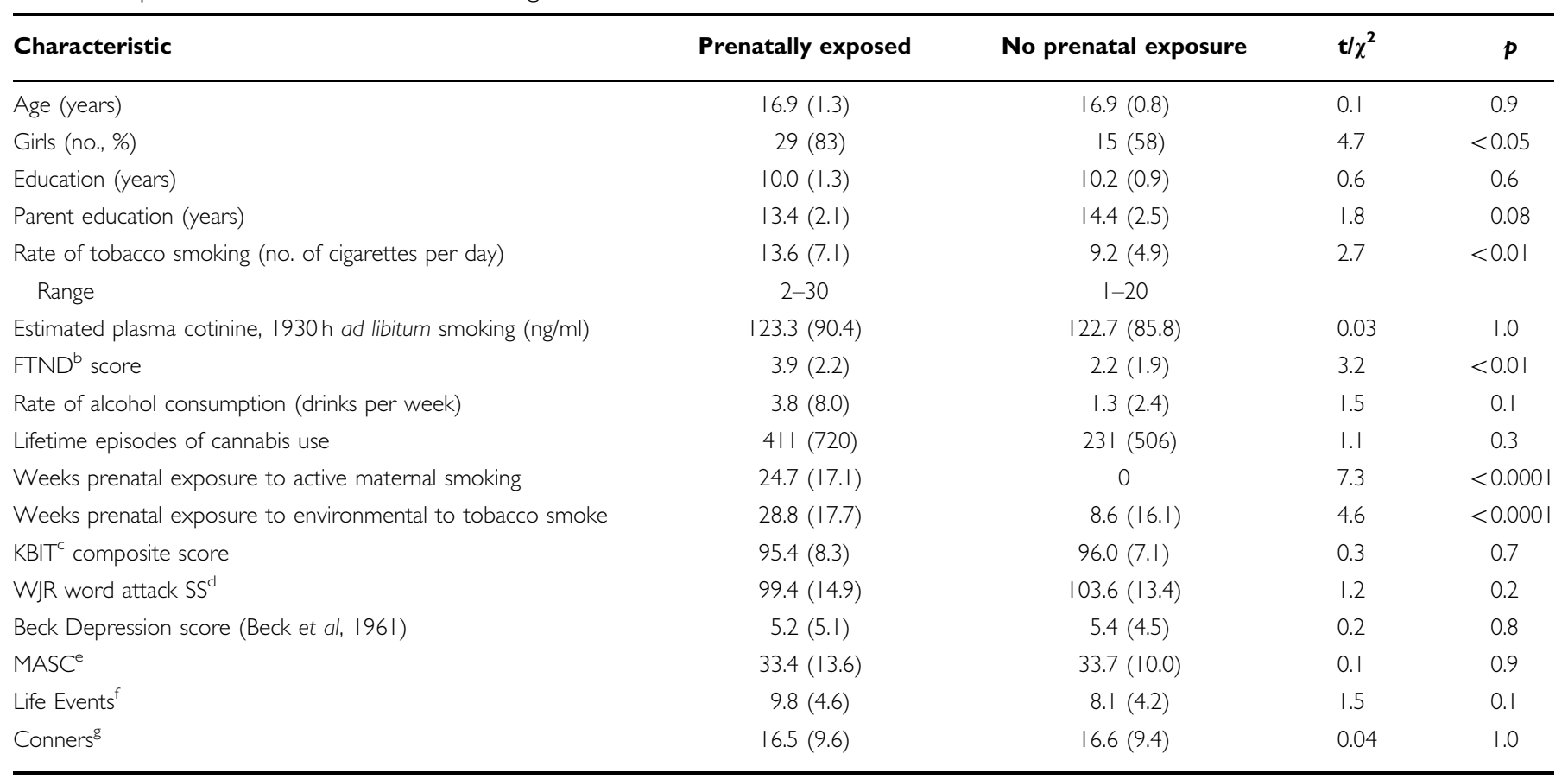

${ }^{a}$ Data are presented as mean (SD) unless otherwise specified.

${ }^{b}$ Fagerstrom Test for Nicotine Dependence (Heatherton et al, 1991).

${ }^{c}$ Kaufman Brief Intelligence Test (Bowers and Pantle, 1998).

Woodcock-Johnson Revised Test of Achievement Word Attack subtest standard scores.

eMultidimensional Anxiety Scale for Children (March et al, 1997).

fLife Events Questionnaire (Masten et al, 1994).

${ }^{8}$ Conners Adolescent Self-Report Scale (Conners, 1998). 
or level of anxiety at the initial screening visit, number of stressful life events, or symptoms of inattention. The prenatally exposed group included more girls and there was a trend for parents of subjects with no prenatal exposure to have received, on average, 1 more year of education than parents of subjects with prenatal exposure. Consistent with previous evidence that maternal smoking during pregnancy increases the risk of smoking in offspring (Buka et al, 2003; Cornelius et al, 2000; Griesler et al, 1998; Kandel et al, 1994), the rate of tobacco smoking and symptoms of nicotine dependence were higher among prenatally exposed adolescent smokers than among adolescents with no prenatal exposure. However, estimated plasma cotinine measured at 7:30 pm during a day of ad libitum smoking did not differ across groups.

Adolescents with prenatal exposure also had more environmental tobacco smoke exposure during gestation (persons other than the mother smoking in the home during gestation of the subject). Among the 35 adolescents with prenatal exposure, exposure was restricted to the first trimester in 16, while 19 were exposed throughout gestation. Reported maternal use of other drugs during pregnancy was as follows: among mothers who smoked tobacco during gestation of the subject, five consumed alcohol during three trimesters, one consumed alcohol during two trimesters, and one consumed alcohol during one trimester. Two of the mothers who consumed alcohol during gestation of the subject also consumed cannabis, one during three trimesters, and one during two trimesters. One mother who consumed alcohol during gestation of the subject also consumed cocaine during three trimesters. Among mothers who did not smoke tobacco during gestation of the subject, two consumed alcohol during three trimesters and none consumed cannabis or cocaine. No subject in either group was exposed to opiates, amphetamine, hallucinogens, sedative-hypnotics, or inhalants during gestation. The proportion of subjects exposed to alcohol during gestation did not significantly differ across groups $\left(\chi^{2}=1.8, p=0.2\right)$, nor did the proportion of subjects exposed to cannabis $\left(\chi^{2}=1.5, p=0.2\right.$; Fisher exact $\left.p=0.5\right)$, or cocaine $\left(\chi^{2}=0.8\right.$, $p=0.4$; Fisher exact $p=1.0$ ) during gestation.

Five subjects with prenatal exposure had experimented with illicit substances other than cannabis; three had tried cocaine, one had tried oral opiates, one had tried sedativehypnotics, and one had tried hallucinogens. Similarly, five subjects with no prenatal exposure had experimented with illicit substances other than cannabis; three had tried cocaine, one had tried amphetamine, one had tried oral opiates, and one had tried hallucinogens. All subjects denied previous use of methylenedioxymethamphetamine (ecstasy), inhalants, anabolic steroids, and injected drugs.

\section{Nicotine Plasma Levels and Effects of Prenatal Exposure and Smoking Condition on Performance}

The average duration between test sessions was $13.5 \pm 12.3$ days and did not differ between groups. Abstinence significantly reduced nicotine, cotinine, and expired air CO (a product of combustion: effect of smoking condition: $\beta=17.3, t=11.5, p<0.0001$ for nicotine, $\beta=58.8, t=4.2$, $p=0.0001$ for cotinine, $\beta=8.4, t=7.5, p<0.0001$ for expired air $\mathrm{CO}$ ), and this effect did not differ between groups. Estimated plasma nicotine concentrations were consistent with compliance with the tobacco restriction prior to the abstinence test session (smoking condition: nicotine $=16.2 \pm 7.6 \mathrm{ng} / \mathrm{ml}$, abstinence: nicotine $=0.5 \pm$ $0.8 \mathrm{ng} / \mathrm{ml}$, range $=0-2.0 \mathrm{ng} / \mathrm{ml}$ ). Consistent with previous reports (Jacobsen et al, 2005), smoking abstinence increased tobacco craving (Tiffany: $\beta=-28.6, t=-4.2, p=0.0001$; Shifman Jarvik Scale: $\beta=-1.6, t=-4.1, p=0.0001)$. The effect of smoking abstinence on symptoms of nicotine withdrawal was marginally significant (Minnesota Nicotine Withdrawal Scale: $\beta=-4.2, t=-1.9, p=0.056)$. Group by smoking condition interaction effects on symptoms of craving and nicotine withdrawal were not significant.

Assessment of visuospatial memory revealed a significant effect of group on BVMT-R total immediate recall ( $\beta=-5.5, t=-3.6, p=0.0008)$, which was modified by smoking condition (group by smoking condition $\beta=3.1$, $t=2.3, p=0.02$; Figure 2a). During ad libitum smoking, prenatally exposed subjects had poorer immediate visuospatial recall than subjects with no prenatal exposure. During the abstinence condition, immediate recall improved in subjects with no prenatal exposure, but worsened in prenatally exposed subjects. Significant group and group by smoking condition interaction effects were also observed for BVMT-R delayed recall. During ad libitum smoking, delayed recall of figures was similar among subjects with and without prenatal exposure. However, during smoking abstinence, visuospatial delayed recall declined in prenatally exposed subjects and improved in subjects with no prenatal exposure (effect of group: $\beta=-1.6, t=2.9, p=0.006$; group by smoking condition interaction effect: $\beta=1.1, t=2.2$, $p=0.03$; Figure $2 \mathrm{~b}$ ). Group and group by smoking condition interaction effects on HVLT-R performance were
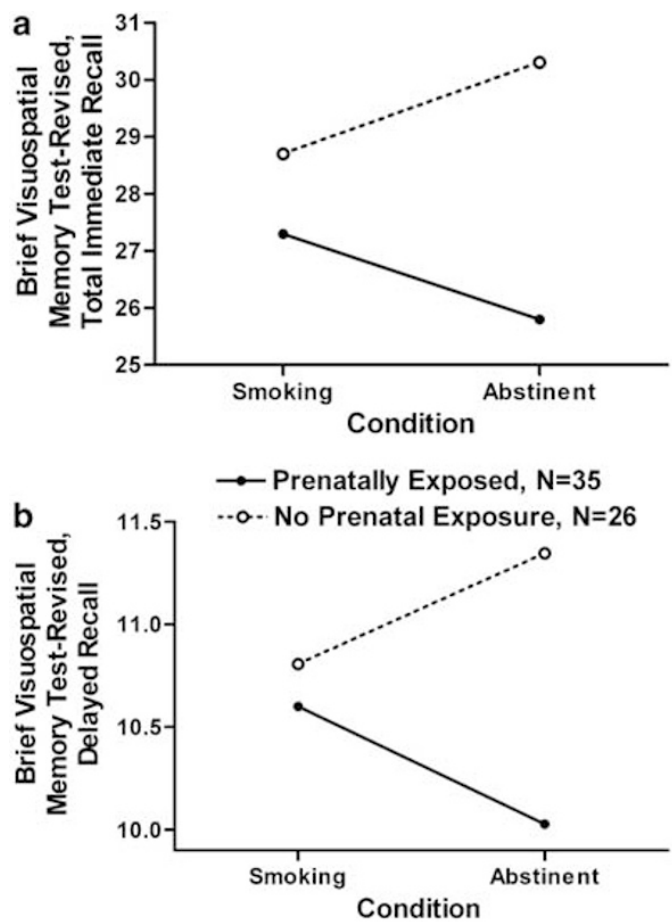

Figure 2 Group by smoking condition interaction effects on BVMT-R total immediate recall (a; $\beta=3.0, t=2.3, p=0.02$ ) and delayed recall ( $b$; $\beta=1.1, t=2.2, p=0.03$ ). 
not significant. Adding the number of lifetime episodes of cannabis use and rate of alcohol consumption by the subjects into these regression models did not change the significance of any of these results.

Examination of the effects of the number of trimesters of exposure revealed no significant differences between subjects exposed to active maternal smoking for one or three trimesters on BVMT-R performance ( $p$-values $>0.4)$.

Within the subgroup of subjects who underwent fMRI scanning, smoking abstinence produced significant declines in plasma nicotine and expired air CO concentrations ( $\beta=13.4, t=4.8, p=0.009$ for plasma nicotine; $\beta=8.5$, $t=3.9, p=0.002$ for expired air CO), and these effects did not differ across groups. Group and group by smoking condition interaction effects on accuracy and speed of task performance during scanning were not significant ( $p$-values $>0.2$ ).

\section{Effects of Prenatal Exposure and Smoking Condition on Task-Related Medial Temporal Lobe Activation}

Given the evidence from BVMT-R performance suggesting that neural circuits subserving visuospatial memory may be rendered vulnerable to the destabilizing effects of nicotine withdrawal by prenatal exposure to active maternal smoking, we focused the analysis of imaging data on identification of medial temporal lobe regions showing significant group by smoking condition interaction effects during mnemonic processing of visuospatial stimuli. Group by smoking condition interaction effects on medial temporal lobe activation during encoding and rehearsal were not significant. A significant group by smoking condition interaction effect was observed in a region of the left parahippocampal gyrus (Talairach coordinates: $X=-17$, $Y=-40, Z=-4$, volume $=72 \mathrm{~mm}^{3}$ ) during testing of early recognition memory for familiar stimuli. Smoking abstinence was associated with increased activation of the left parahippocampal gyrus in subjects with prenatal exposure and decreased activation of this structure in subjects with no prenatal exposure.

Significant group by smoking condition interaction effects were observed at the left hippocampus $(X=-29$, $Y=-29, Z=-7)$, the lateral aspect of the right hippocampus $(X=38, Y=-23, Z=-7)$, and the left insular cortex $(X=-33, Y=4, Z=-7)$ during testing of delayed recognition memory for familiar stimuli. These results are presented in Figure 3. Linear contrasts were applied to identify regions that increase in activity during nicotine withdrawal to a greater degree in subjects with prenatal exposure relative to subjects with no prenatal exposure as red/yellow. Regions demonstrating the opposite pattern (decreasing in activity during nicotine withdrawal to a greater degree in subjects with prenatal exposure relative to subjects with no prenatal exposure) are shown in blue/ purple. Data are displayed per radiological convention, with the right side of the brain on the left side of the figure. Average percent signal change of the left and right hippocampal voxels demonstrating significant group by smoking condition interaction effects are plotted for each group and smoking condition. During testing of delayed recognition memory, smoking abstinence was associated with increased activation of the left and right hippocampus

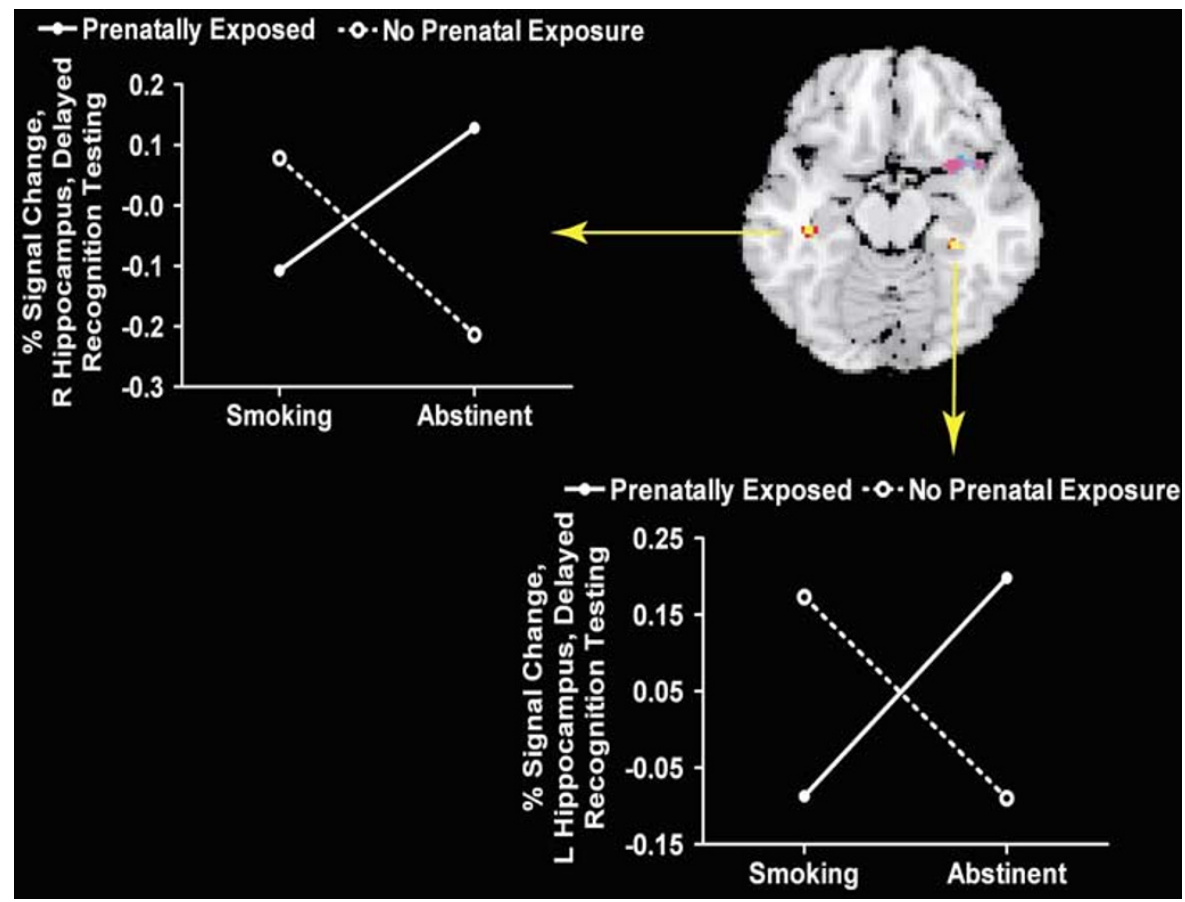

Figure 3 Group by smoking condition interaction effects on activation of the left and right hippocampus during delayed recognition testing of visuospatial stimuli. Images are displayed per radiological convention, with the right side of the brain on the left side of the figure. Linear contrasts were applied to identify regions where prenatally exposed subjects show significant increases in activity relative to controls during smoking abstinence as red/yellow. Regions demonstrating the opposite pattern (significantly decreased activation during smoking abstinence in prenatally exposed subjects relative to controls) are shown in blue/purple. Voxel threshold: $p<0.00 \mathrm{I}$, cluster threshold: eight contiguous significant voxels. Plots are shown of the average percent signal change for the left and right hippocampal voxels demonstrating significant group by smoking condition interaction effects. 
in subjects with prenatal exposure and decreased activation of these structures in subjects with no prenatal exposure.

\section{DISCUSSION}

In the present study, adolescent tobacco smokers with prenatal exposure to active maternal smoking experienced greater nicotine withdrawal-related deficits in immediate and delayed visuospatial memory relative to adolescent smokers with no prenatal exposure to maternal smoking. Among adolescent smokers with prenatal exposure, nicotine withdrawal was associated with increased activation of the left parahippocampal gyrus during early recognition testing of visuospatial stimuli and increased activation of bilateral hippocampus during delayed recognition testing of visuospatial stimuli. These findings are consistent with prior work in animals and in humans, indicating that gestational exposure to nicotine absorbed during active maternal smoking exerts long-lasting effects on brain development that alter the subsequent brain response to nicotine during adolescence (Kirkland et al, 2000; Cornelius et al, 2000; Griesler et al, 1998; Kandel et al, 1994; AbreuVillaca et al, 2004a, b).

Work in rodents has shown that combining prenatal with adolescent exposure to nicotine produces additive effects on neurons and synaptic function (Abreu-Villaca et al, 2004a, b). Prenatal nicotine exposure increases hippocampal size, DNA content, and neuronal packing density (Roy et al, 2002; Abreu-Villaca et al, 2004a). The hippocampus is unique among brain regions in retaining the capacity to generate new neurons into adolescence (McEwen, 1999, 2001), and has been shown to exhibit synaptic sprouting and reinnnervation in response to lesioning throughout the juvenile period (McWilliams and Lynch, 1983, 1984). Hippocampal changes pursuant to gestational exposure to nicotine thus likely reflect adaptive rearrangement of neuronal populations and synapses (Abreu-Villaca et al, 2004a), and are magnified when followed by adolescent nicotine administration (Abreu-Villaca et al, 2004a). The present findings suggest that, in human adolescents, changes in hippocampal architecture associated with combined prenatal and adolescent exposure to active tobacco smoking produce alterations in hippocampal function during nicotine withdrawal and concomitant deficits in visuospatial memory.

Animal studies have also demonstrated evidence that prenatal exposure to nicotine impairs cholinergic responsiveness in adolescence, blunting the ability of nicotine to upregulate nAChRs and desensitizing neuronal responses to nicotine challenge (Abreu-Villaca et al, 2004b; Seidler et al, 1992). In addition, nicotine withdrawal has been found to induce cholinergic hypoactivity in adolescent rodents with prenatal exposure to nicotine (Abreu-Villaca et al, 2004b). Given that reductions in cholinergic neurotransmission disrupt memory in humans (Rosier et al, 1998, 1999; Mewaldt and Ghoneim, 1979), the present observations of significant decrements in visuospatial memory that emerge during nicotine withdrawal in adolescent smokers with exposure to active maternal smoking are consistent with these preclinical findings. In the present study, selfadministration of nicotine via tobacco smoking was associated with a reversal of withdrawal-related deficits in memory function in prenatally exposed adolescents. Cholinergic hypoactivity induced by nicotine withdrawal in prenatally exposed adolescent rodents is prolonged (AbreuVillaca et al, 2004a). Together, these observations lend strength to the proposal that cholinergic hypoactivity may be a mechanism by which prenatal exposure elevates the risk of regular tobacco smoking, nicotine dependence, and relapse to smoking during quit attempts in offspring, as nicotine may be taken to self-medicate brain functional deficits resulting from cholinergic hypoactivity (AbreuVillaca et al, 2004a). Reduced cholinergic reactivity and nicotine response in adolescents with prenatal exposure would also drive higher tobacco consumption in order to achieve the requisite restoration of 'normal' cholinergic function, and thus may have contributed to the higher rate of cigarette consumption observed in the present sample of prenatally exposed adolescents and in other samples of prenatally exposed adults (Buka et al, 2003). Conversely, cognitive deficits experienced by adolescent smokers with prenatal exposure to maternal smoking in school, where smoking is not permitted, may place them at greater risk of academic failure.

In the present study, BVMT-R total immediate and delayed recall tended to improve during smoking abstinence in adolescents with no prenatal exposure to active maternal smoking. Nicotinic receptors desensitize following exposure to nicotine, with desensitization occurring over seconds and resensitization occurring over minutes (Grady et al, 1994). Work in rats has shown that nicotine administration induces upregulation of nAChRs (Nguyen et al, 2003), while postmortem work in humans has shown that tobacco smoking increases nAChR-binding sites in adult smokers in a dose-dependent manner (Perry et al, 1999). During smoking abstinence, these subjects have an increased number of sensitized $\mathrm{nAChR}$ receptors available to endogenous acetylcholine (Leonard and Giordano, 2002). Enhancement of cholinergic neurotransmission has been shown to improve memory performance by improving the selectivity of perceptual processing during encoding (Furey et al, 2000). Two prior studies have observed improved memory performance in healthy adult smokers during acute smoking abstinence (George et al, 2002; Jacobsen et al, 2004). The present findings suggest that this effect occurs in adolescent smokers and is modified by prenatal exposure to active maternal smoking.

Prior work in rodents using tone-shock fear conditioning and the Hebb-Williams maze has provided evidence that reduction of cholinergic neurotransmission is disruptive to encoding, but not to retrieval (Rogers and Kesner, 2003, 2004). In the present study, significant group differences in modulation of hippocampal and parahippocampal activation by smoking condition were evident only during early and delayed recognition testing and not during encoding or rehearsal. Increased hippocampal and parahippocampal activation observed during smoking abstinence in prenatally exposed adolescents during recognition testing may reflect compensatory activation in response to deficient encoding. Supporting this interpretation, prior work has shown that enhanced cholinergic neurotransmission increases the activation of primary sensory cortices during encoding and reduces the activation of brain regions 
involved in higher-order processing during mnemonic tasks (Furey et al, 2000). This finding suggests that increased cholinergic neurotransmission enhances sensory processing during encoding, thereby reducing the demand on brain regions that mediate higher-order mnemonic processes (Furey et al, 2000). If, as suggested by preclinical and postmortem studies reviewed above, smoking abstinence leads to an increase in sensitized $n A C h R$ receptors and cholinergic neurotransmission in adolescent smokers without prenatal exposure to active maternal smoking, then reduced medial temporal lobe activation observed in these subjects during delayed recognition testing during smoking abstinence may stem from cholinergically mediated enhancements in encoding, leading to reduced hippocampal and parahippocampal load during recognition testing. Conversely, if prenatally exposed adolescent smokers do not upregulate nAChRs during smoking and thus experience decreased cholinergic neurotransmission during smoking abstinence, then increased medial temporal lobe activation observed in these subjects during delayed recognition testing during smoking abstinence may stem from less effective encoding, leading to increased hippocampal and parahippocampal load. Our failure to observe significant group differences in the effect of smoking cessation on medial temporal lobe activation during encoding may stem from the limited size of the sample undergoing imaging in the present study. Accordingly, findings regarding the effect of prenatal exposure to active maternal smoking on changes in hippocampal and parahippocampal function induced by smoking cessation in adolescents during mnemonic processing must be viewed as preliminary until replicated.

The possibility that the group differences in cognitive performance and brain function observed in this study stem from factors unrelated to prenatal exposure to active maternal smoking cannot be excluded by the present data. However, the fact that group by smoking condition interaction effects remained significant after group matching and statistical controls for potentially confounding variables, including cannabis and alcohol use, argues against this possibility, as does the resemblance of our findings to the effects predicted from animal models of prenatal and adolescent nicotine exposure and withdrawal. More females than males participated in this preliminary study. Preclinical work has suggested that the combined effects of prenatal and adolescent exposure to nicotine on protein biomarkers may be more marked in female rodents (Abreu-Villaca et al, 2004a), while the combined effects of prenatal and adolescent exposure on some markers of cholinergic tone may be more pronounced in male rodents (Abreu-Villaca et al, 2004b). Recent clinical work has suggested that the effects of adolescent tobacco smoking on cognitive performance may be more pronounced in males (Jacobsen et al, 2005). Thus, the effect of gender on the vulnerability of the brain to the combined effects of prenatal and adolescent nicotine exposure is likely complicated. Although the findings observed in the present study remained significant after statistically controlling for the differences in gender distribution across groups, further work is needed in larger samples balanced for gender in order to determine how gender may modify the effects of combined prenatal and adolescent exposure to tobacco smoking on brain function. An effect of prenatal exposure on verbal memory was not detected in this study. However, the possibility that the divergent findings regarding effects of prenatal exposure on verbal and visuospatial memory stem from subtle differences in the difficulty of the HVLT-R and BVMT-R tasks, as opposed to differences in the vulnerability of neural systems supporting verbal and visuospatial memory to damage resulting from prenatal exposure to nicotine, cannot be excluded by the present data. Other limitations include the measurement of prenatal exposure by retrospective self-report. Work comparing prospectively and retrospectively collected data about pregnancy has supported the accuracy of pregnancy data collected by retrospective self-report (Buka et al, 2004). Similarly, comparison of serum cotinine concentrations and self-reported smoking behavior during pregnancy has yielded evidence of significant agreement between these measures (Buka et al, 2003). Nevertheless, rates of smoking during pregnancy may have been underreported in the present study due to the social stigma attached to this behavior. Underreporting of maternal smoking during pregnancy in the unexposed group would tend to reduce the observed group differences. Thus, to the extent that this occurred, the actual effects of gestational exposure to active maternal smoking on brain response to smoking and nicotine withdrawal during adolescence may be larger than those reported herein. Finally, the possibility that genetic factors shared by mothers and their offspring may mediate both increased risk for smoking and increased vulnerability to the development of memory deficits during nicotine withdrawal cannot be excluded by these data.

In summary, the present results indicate that adolescent smokers exposed to active maternal smoking during gestation experience greater abstinence-induced deficits in visuospatial memory and concomitant changes in hippocampal and parahippocampal function relative to adolescent smokers with no gestational exposure to active maternal smoking. These findings are consistent with preclinical data showing that combined prenatal and adolescent exposure to nicotine produces reductions in cholinergic neurotransmission and more pronounced changes in hippocampal function than does either prenatal or adolescent exposure alone (Abreu-Villaca et al, 2004a,b). At present, approximately $11 \%$ of women worldwide who give birth are unable to stop smoking during their pregnancies (Martin et al, 2005; Windsor, 2001). The present findings underscore the importance of developing more effective treatments for smoking cessation that do not involve nicotine replacement for these women. Equally critical, the fact that exposure limited to the first trimester and exposure throughout gestation produced similar effects emphasizes the need to focus treatment efforts on women of childbearing age, in addition to pregnant women. Further, these data extend previous work linking prenatal exposure to active maternal smoking with elevated risk of subsequent tobacco smoking and nicotine dependence, and suggest that prenatal exposure may define a subgroup of adolescent smokers who may particularly benefit from smoking cessation treatment that addresses cessation-related changes in brain function. Further work is needed to develop therapies designed to reduce the functional deficits associated with smoking abstinence in this population of adolescents. 


\section{ACKNOWLEDGEMENTS}

This work was supported by United States Public Health Service grants DA14655, DA017333, and MO1 RR000125. We thank Dr Jonathan S Feinstein for statistical consultation and the subjects and their families for their participation.

\section{REFERENCES}

Abreu-Villaca Y, Seidler FJ, Qiao D, Tate CA, Cousins MM, Thillai I et al (2003). Short-term adolescent nicotine exposure has immediate and persistent effects on cholinergic systems: critical periods, patterns of exposure, dose thresholds. Neuropsychopharmacology 28: 1935-1949.

Abreu-Villaca Y, Seidler FJ, Slotkin TA (2004a). Does prenatal nicotine exposure sensitize the brain to nicotine-induced neurotoxicity in adolescence? Neuropsychopharmacology 29: $1440-1450$.

Abreu-Villaca Y, Seidler FJ, Tate CA, Cousins MM, Slotkin TA (2004b). Prenatal nicotine exposure alters the response to nicotine administration in adolescence: effects on cholinergic systems during exposure and withdrawal. Neuropsychopharmacology 29: 879-890.

Atluri P, Fleck MW, Shen Q, Mah SJ, Stadfelt D, Barnes W et al (2001). Functional nicotinic acetylcholine receptor expression in stem and progenitor cells for the early embryonic mouse cerebral cortex. Dev Biol 240: 143-156.

Bayer SA (1983). [ $\left.{ }^{3} \mathrm{H}\right]$ thymidine-radiographic studies of neurogenesis in the rat olfactory bulb. Exp Brain Res 50: 329-340.

Bayer SA, Yackel JW, Puri PS (1982). Neurons in the rat dentate gyrus granular layer substantially increase during juvenile and adult life. Science 216: 890-892.

Beck AT, Ward CH, Mendelson M, Mock J, Erbaugh J (1961). An inventory for measuring depression. Arch Gen Psychiatry 4: 561-571.

Benedict RHB, Schretlen D, Groninger L, Dobraski M, Shpritz B (1996). Revision of the brief visuospatial memory test: studies of normal performance, reliability, and validity. Psychol Assess 8: $145-153$

Benedict RHB, Zgaljardic DJ (1998). Practice effects during repeated administrations of memory tests with and without alternate forms. J Clin Exp Neuropsychol 20: 339-352.

Bowers TL, Pantle ML (1998). Shipley institute for living scale and the Kaufman Brief Intelligence Test as screening instruments for intelligence. Assessment 5: 187-195.

Boynton GM, Engel SA, Glover GH, Heeger DJ (1996). Linear systems analysis of functional magnetic resonance imaging in human VI. J Neurosci 16: 4207-4221.

Breslau N, Johnson EO, Hiripi E, Kessler R (2001). Nicotine dependence in the United States. Arch Gen Psychiatry 58: 810-816.

Buka SL, Goldstein JM, Spartos E, Tsuang MT (2004). The retrospective measurement of prenatal and perinatal events: accuracy of maternal recall. Schizophr Res 71: 417-426.

Buka SL, Shenassa ED, Niaura R (2003). Elevated risk of tobacco dependence among offspring of mothers who smoked during pregnancy: a 30-year prospective study. Am J Psychiatry 160: 1978-1984.

Cabeza R, Rao SM, Wagner AD, Mayer AR, Schacter DL (2001). Can medial temporal lobe regions distinguish true from false? An event-related functional MRI study of veridical and illusory recognition memory. Proc Natl Acad Sci USA 98: $4805-4810$.

Conners CK (1998). Rating scales in attention-deficit/hyperactivity disorder: use in assessment and treatment monitoring. J Clin Psychiatry 59(Suppl 7): 24-30.
Cornelius MD, Leech SL, Goldschmidt L, Day N (2000). Prenatal tobacco exposure: is it a risk factor for early tobacco experimentation? Nicotine Tobacco Res 2: 45-52.

Cornelius MD, Ryan CM, Day NL, Goldschmidt L, Willford JA (2001). Prenatal tobacco effects on neuropsychological outcomes among preadolescents. J Dev Behav Pediatr 22: 217-225.

Donaldson DI, Petersen SE, Ollinger JM, Buckner RL (2001). Dissociating state and item components of recognition memory using fMRI. Neuroimage 13: 129-142.

Duncan JS, Papademetris X, Yang J, Jackowski M, Zeng X, Staib LH (2004). Geometric strategies for neuroanatomic analysis from MRI. Neuroimage 23(Suppl 1): 534-545.

Ebrahim SH, Floyd RL, Merritt RK, Decoufle P, Holtzman D (2000). Trends in pregnancy-related smoking rates in the United States, 1987-1996. JAMA 283: 361-366.

Eichenbaum H, Schoenbaum G, Young B, Bunsey M (1996). Functional organization of the hippocampal memory system. Proc Natl Acad Sci USA 93: 13500-13507.

Foulds J, Stapleton J, Swettenham J, Bell N, McSorley K, Russell MAH (1996). Cognitive performance effects of subcutaneous nicotine in smokers and never-smokers. Psychopharmacology 127: $31-38$.

Fried PA, O'Connell CM, Watkinson B (1992). 60- and 72-month follow-up of children prenatally exposed to marijuana, cigarettes and alcohol: cognitive and language assessment. J Dev Behav Pediatr 13: 383-391.

Fried PA, Watkinson B (1990). 36- and 48-month neurobehavioral follow-up of children prenatally exposed to marijuana, cigarettes and alcohol. J Dev Behav Pediatr 11: 49-58.

Fried PA, Watkinson B, Gray R (1998). Differential effects on cognitive functioning in 9- to 12-year olds prenatally exposed to cigarettes and marihuana. Neurotoxicol Teratol 20: 293-306.

Fried PA, Watkinson B, Gray R (2003). Differential effects on cognitive functioning in 13- to 16-year-olds prenatally exposed to cigarettes and marihuana. Neurotoxicol Teratol 25: 427-436.

Friston KJ, Ashburner J, Frith CD, Poline JB, Heather JD, Frackowiak RS (1995). Spatial registration and normalization of images. Hum Brain Map 2: 165-189.

Furey ML, Pietrini P, Haxby JV (2000). Cholinergic enhancement and increased selectivity of perceptual processing during working memory. Science 290: 2315-2319.

George TP, Vessicchio JC, Termine A, Sahady DM, Head CA, Pepper WT et al (2002). Effects of smoking abstinence on visuospatial working memory function in schizophrenia. Neuropsychopharmacology 26: 75-85.

Grady SR, Marks MJ, Collins AC (1994). Desensitization of nicotine-stimulated $\left[{ }^{3} \mathrm{H}\right]$ dopamine release from mouse striatal synaptosomes. J Neurochem 62: 1390-1398.

Griesler PC, Kandel DB, Davies M (1998). Maternal smoking in pregnancy, child behavior problems, and adolescent smoking. J Res Adolesc 8: 159-185.

Gusella JL, Fried PA (1984). Effects of maternal social drinking and smoking on offspring at 13 months. Neurobehav Toxicol Teratol 6: 13-17.

Heatherton TF, Kozlowski LT, Frecker RC, Fagerstrom KO (1991). The Fagerstrom test for nicotine dependence: a revision of the Fagerstrom Tolerance Questionnaire. Br J Addict 86: 1119-1127.

Holmes AP, Friston KJ (1998). Generalizability, random effects, and population inference. Neuroimage 7: S34.

Hughes J, Hatsukami DK (1998). Errors in using tobacco withdrawal scale. Tobacco Control 7: 92-93.

Huttenlocher PR (1990). Morphometric study of human cerebral cortex development. Neuropsychologia 28: 517-527.

Jacob P, Wilson M, Benowitz NL (1981). Improved gas chromatographic method for the determination of nicotine and cotinine in biologic fluids. J Chromatogr 222: 61-70.

Jacobsen LK, D'Souza DC, Mencl WE, Pugh KR, Skudlarski P, Krystal JH (2004). Nicotine effects on brain function and 
functional connectivity in schizophrenia. Biol Psychiatry 55: 850-858.

Jacobsen LK, Krystal JH, Mencl WE, Westerveld M, Frost SJ, Pugh KR (2005). Effects of smoking and smoking abstinence on cognition in adolescent tobacco smokers. Biol Psychiatry 57: $56-66$.

Jarvis MJ, Primatesta P, Erens B, Feyerabend C, Bryant A (2003). Measuring nicotine intake in population surveys: comparability of saliva cotinine and plasma cotinine estimates. Nicotine Tobacco Res 5: 349-355.

Kandel DB, Wu P, Davies P (1994). Maternal smoking during pregnancy and smoking by adolescent daughters. Am J Public Health 84: 1407-1413.

Kaufman J, Birmaher B, Brent D, Rao U, Ryan N (1996). The Schedule for Affective Disorders and Schizophrenia for School Aged Children: Present and Lifetime Version. Western Psychiatric Institute and Clinic, University of Pittsburgh: Pittsburgh.

Kirk RE (1982). Experimental Design: Procedures for the Social Sciences. Wadsworth: Belmont, CA.

Kirkland SA, Dodds LA, Brosky G (2000). The natural history of smoking during pregnancy among women in Nova Scotia. Canad Med Assoc J 163: 281-282.

Krebs SJ, Petros TV, Beckwith BE (1994). Effects of smoking on memory for prose passages. Physiol Behav 56: 723-727.

Lauder JM, Schambra UB (1999). Morphogenetic roles of acetylcholine. Environ Health Persp 107(Suppl 1): 65-69.

Leonard S, Giordano L (2002). Are differential behavioral responses to smoking and smoking cessation in schizophrenia related to nicotinic receptor levels? Neuropsychopharmacology 27: 1082-1083.

Levin ED, Briggs SJ, Christopher NC, Rose JE (1993). Prenatal nicotine exposure and cognitive performance in rats. Neurotoxicol Teratol 15: 251-260.

Levin ED, Wilkerson A, Jones JP, Christopher NC, Briggs SJ (1996). Prenatal nicotine effects on memory in rats: pharmacological and behavioral challenges. Brain Res Dev Brain Res 97: 207-215.

March JS, Parker JD, Sullivan K, Stallings P, Conners CK (1997). The Multidimensional Anxiety Scale for Children (MASC): factor structure, reliability, and validity. J Am Acad Child Adolesc Psychiatry 36: 554-565.

Martin JA, Kochanek KD, Strobino DM, Guyer B, MacDorman MF (2005). Annual summary of vital statistics - 2003. Pediatrics 115: 619-634.

Masten AS, Neemann J, Andenas S (1994). Life events and adjustment in adolescents: the significance of event independence, desirability, and chronicity. J Res Adolesc 4: 71-97.

Matthews DA, Nadler JV, Lynch GS, Cotman CW (1974). Development of cholinergic innervation in the hippocampal formation of the rat. Dev Biol 36: 130-141.

McEwen BS (1999). Stress and hippocampal plasticity. Annu Rev Neurosci 22: 105-122.

McEwen BS (2001). Plasticity of the hippocampus: adaptation to chronic stress and allostatic load. Ann NY Acad Sci 933: 265-277.

McWilliams JR, Lynch G (1983). Rate of synaptic replacement in denervated rat hippocampus declines precipitously from the juvenile period to adulthood. Science 221: 572-574.

McWilliams JR, Lynch G (1984). Synaptic density and axonal sprouting in rat hippocampus: stability in adulthood and decline in late adulthood. Brain Res 294: 152-156.

Mencl WE, Pugh KR, Shaywitz SE, Shaywitz BA, Fulbright RK, Constable RT et al (2000). Network analysis of brain activation in working memory: behavior and age relationships. Microsc Res Techn 51: 64-74.

Mewaldt SP, Ghoneim MM (1979). The effects and interactions of scopolamine, physostigmine and methamphetamine on human memory. Pharmacol Biochem Behav 10: 205-210.

Meyers K, McLellan AT, Jaeger JL, Pettinati HM (1995). The development of the comprehensive addiction severity index for adolescents (CASI-A). An interview for assessing multiple problems of adolescents. J Subst Abuse Treat 12: 181-193.

Nadler JV, Matthews DA, Cotman CW, Lynch GS (1974). Development of cholinergic innervation in the hippocampal formation of the rat: II. Quantitative changes in choline acetyltransferase and acetylcholinesterase activities. Dev Biol 36: $142-154$.

Navarro HA, Seidler FJ, Eylers JP, Baker FE, Dobbins SS, Lappi SE et al (1989). Effects of prenatal nicotine exposure on development of central and peripheral cholinergic neurotransmitter systems. Evidence for cholinergic trophic influences in developing brain. J Pharmacol Exp Ther 251: 894-900.

Navarro HA, Seidler FJ, Whitmore WL, Slotkin TA (1988). Prenatal exposure to nicotine via maternal infusions: effects on development of catecholamine systems. J Pharmacol Exp Ther 244: 940-944.

Nguyen HN, Rasmussen BA, Perry DC (2003). Subtype-selective up-regulation by chronic nicotine of high affinity nicotinic receptors in rat brain demonstrated by receptor autoradiography. J Pharmacol Exp Ther 307: 1090-1097.

Otten LJ, Henson RNA, Rugg MD (2002). State-related and itemrelated neural correlates of successful memory encoding. Nat Neurosci 5: 1339-1344.

Perry DC, Davila-Garcia MI, Stockmeier CA, Kellar KJ (1999). Increased nicotinic receptors in brains from smokers: membrane binding and autoradiography studies. J Pharmacol Exp Ther 289: $1545-1552$.

Pineda JA, Herrera C, Kang C, Sandler A (1998). Effects of cigarette smoking and 12-h abstention on working memory during a serial-probe recognition task. Psychopharmacology 139: 311-321.

Rogers JL, Kesner RP (2003). Cholinergic modulation of the hippocampus during encoding and retrieval. Neurobiol Learn Memory 80: 332-342.

Rogers JL, Kesner RP (2004). Cholinergic modulation of the hippocampus during encoding and retrieval of tone/shockinduced fear conditioning. Learn Memory 11: 102-107.

Rosier A, Cornette L, Orban GA (1998). Scopolamine-induced impairment of delayed recognition of abstract visual shapes. Neuropsychobiology 37: 98-103.

Rosier AM, Cornette L, Dupont P, Bormans G, Mortelmans L, Orban GA (1999). Regional brain activity during shape recognition impaired by a scopolamine challenge to encoding. Eur J Neurosci 11: 3701-3714.

Roy TS, Andrews JE, Seidler FJ, Slotkin TA (1998). Nicotine evokes cell death in embryonic rat brain during neurulation. J Pharmacol Exp Ther 287: 1136-1144.

Roy TS, Seidler FJ, Slotkin TA (2002). Prenatal nicotine exposure evokes alterations of cell structure in hippocampus and somatosensory cortex. J Pharmacol Exp Ther 300: 124-133.

Schneider AS, Atluri P, Shen Q, Barnes W, Mah SJ, Stadfelt D et al (2002). Functional nicotinic acetylcholine receptor expression on stem and progenitor cells of the early embryonic nervous system. Ann NY Acad Sci 971: 135-138.

Seidler FJ, Levin ED, Lappi SE, Slotkin TA (1992). Fetal nicotine exposure ablates the ability of postnatal nicotine challenge to release norepinephrine from rat brain regions. Brain Res Dev Brain Res 69: 288-291.

Shapiro AM, Benedict RH, Schretlen D, Brandt J (1999). Construct and concurrent validity of the Hopkins Verbal Learning Testrevised. Clin Neuropsychol 13: 348-358.

Shaywitz SE, Shaywitz BA, Pugh KR, Fulbright RK, Skudlarski P, Mencl WE et al (1999). Effects of estrogen on brain activation patterns in postmenopausal women during working memory tasks. JAMA 281: 1197-1202.

Shiffman S, Paty JA, Gnys M, Kassel JD, Elash C (1995). Nicotine withdrawal in chippers and regular smokers: subjective and cognitive effects. Healthy Psychol 14: 301-309. 
Shiffman SM, Jarvik ME (1976). Smoking withdrawal symptoms in two weeks of abstinence. Psychopharmacology 50: 35-39.

Slotkin TA (1998). Fetal nicotine or cocaine exposure: which one is worse? J Pharmacol Exp Ther 285: 931-945.

Slotkin TA (1999). Developmental cholinotoxicants: nicotine and chlorpyrifos. Environ Health Perspect 107(Suppl 1): 71-80.

Slotkin TA (2002). Nicotine and the adolescent brain: insights from and animal model. Neurotoxicol Teratol 24: 369-384.

Slotkin TA (2004). Cholinergic systems in brain development and disruption by neurotoxicants: nicotine, environmental tobacco smoke, organophosphates. Toxicol Appl Pharmacol 198: 132-151.

Snyder FR, Davis FC, Henningfield JE (1989). The tobacco withdrawal syndrome: performance decrements assessed on a computerized test battery. Drug Alcohol Depend 23: 259-266.

Sperling R, Greve D, Dale A, Killiany R, Holmes J, Rosas HD et al (2002). Functional MRI detection of pharmacologically induced memory impairment. Proc Natl Acad Sci USA 99: 455-460.

Squire LR (1992). Memory and the hippocampus: a synthesis from findings with rats, monkeys, and humans. Psychol Rev 99: 195-231.

Teneggi V, Squassante L, Iavarone L, Milleri S, Bye A, Gomeni R (2002). Correlation and predictive performances of saliva and plasma nicotine concentration on tobacco withdrawal-induced craving. Br J Clin Pharmacol 54: 407-414.

Tiffany ST, Drobes DJ (1991). The development and initial validation of a questionnaire on smoking urges. $\mathrm{Br}$ J Addict 86: 1467-1476.

Trauth JA, Seidler FJ, Ali SF, Slotkin TA (2001). Adolescent nicotine exposure produces immediate and long-term changes in
CNS noradrenergic and dopaminergic function. Brain Res 892: 269-280.

Trauth JA, Seidler FJ, Slotkin TA (2000). An animal model of adolescent nicotine exposure: effects on gene expression and macromolecular constituents in rat brain regions. Brain Res 867: 29-39.

Visscher KM, Miezin FM, Kelly JE, Buckner RL, Donaldson DI, McAvoy MP et al (2003). Mixed blocked/event-related designs separate transient and sustained activity in fMRI. Neuroimage 19: 1694-1708.

Weiss ER, Maness P, Lauder JM (1998). Why do neurotransmitters act like growth factors? Perspect Dev Neurobiol 5: 323-335.

West R, Hack S (1991). Effect of cigarettes on memory search and subjective ratings. Pharmacol Biochem Behav 38: 281-286.

Windsor RA (2001). Smoking cessation and pregnancy. In: Samet JM, Yoon SY (eds). Women and the Tobacco Epidemic: Challenges for the 21st Century. World Health Organization: Geneva, pp 147-164.

Woods RP (1996). Modeling for intergroup comparisons of imaging data. Neuroimage 4: S84-S94.

Zahalka E, Seidler FJ, Lappi SE, Yanai J, Slotkin TA (1993). Differential development of cholinergic nerve terminal markers in rat brain regions: implications for nerve terminal density, impulse activity and specific gene expression. Brain Res 601: 221-229.

Zahalka EA, Seidler FJ, Lappi SE, McCook EC, Yanai J, Slotkin TA (1992). Deficits in development of central cholinergic pathways caused by fetal nicotine exposure: differential effects on choline acetyltransferase activity and $\left[{ }^{3} \mathrm{H}\right]$ hemicholinium- 3 binding. Neurotoxicol Teratol 14: 375-382. 